\title{
Management of Massive Blood Transfusion-a case study
}

\author{
Sufia Khatun Lima ${ }^{1}$, Monowara Begum², Anil Kumar Gupta ${ }^{3}$ Lutful Aziz, ${ }^{4}$ S P Mitra ${ }^{5}$ \\ Summary
}

Management of a complicated obstetric patient with profuse bleeding following caesarean section (under GA) required massive blood transfusion was managed properly with multidisciplinary approach in ICU. The involvement of obstetrician, anesthesiologist, intensivist, general surgeon, hematologist \& gastroenterologist as a team in a single setup is essential for the management of such patients and the best outcome. The patient received total 117 units of blood products among which 20 units whole blood, 17 units packed red blood cell, 40 units of fresh frozen plasma and 40 units of platelet concentrate. Despite this massive blood transfusion, the patient recovered fully with minimal complications as we follow the near standard blood transfusion protocol.

\section{Key words}

Post-operative bleeding, massive blood transfusion, complications, management.

\section{Introduction}

Massive blood transfusion is used for the treatment of uncontrolled hemorrhage. The most common reasons for Massive Transfusion (MT) is trauma ${ }^{1}$ patients with GI bleeding ${ }^{2-4}$ and surgical patients undergoing elective or emergent complex surgical procedures (like abdominal aortic aneurysm repair, orthotopic liver transplantation, major orthopedics surgery). ${ }^{5-7}$ Other causes include cardiac \& vascular surgery, ectopic pregnancy, major obstetric hemorrhage and post-partum uterine bleeding. In the management of acute hemorrhage and hemorrhagic shock, the first priority is to stop hemorrhage and the second, albeit concurrent, priority is blood transfusion. The goal of transfusion of blood and blood products is effective restoration of patient's blood composition within safe limits with regard to haemostasis, oxygen-carrying capacity, oncotic pressure and biochemistry. MT is commonly defined as the administration of $>10$ units of packed RBCs (PRBCs) in 24 hours ${ }_{8,9}$ to an individual patient or the transfusion of more than one blood volume in 24 hours (adult blood volume is approx $70 \mathrm{ml} / \mathrm{kg}) .^{10,11} \mathrm{In}$ children, it is defined as transfusion of $>40 \mathrm{ml} / \mathrm{kg}$. MT protocols have been advocated a
1:1:1 ratio of packed RBCs to fresh frozen plasma to platelet concentrations to avoid dilutional and consumptive coagulopathy and thrombocytopenia.Gonzalez et al. ${ }^{12}$

\section{Case Report}

Ms. Hashi 24 yrs young lady $3^{\text {rd }}$ gravida, para-1(c/s) + 1 abortion, admitted in AHD through ER on 26/11/2011 as a referred patient from another hospital with the diagnosis of 35 completed weeks pregnancy with severe jaundice for safe confinement. Her early part of pregnancy was fine but from 24 weeks of pregnancy she developed jaundice with nausea, vomiting and intermittent fever. On advice from her obstetrician, she consulted with an internist (outside AHD). Investigations showed negative viral markers and was managed conservatively up to 35 weeks. On 26/11/11, she was planned for elective C-section at $10 \mathrm{pm}$ outside AHD. But that was postponed due to the apprehension of per-operative bleeding hazard (outside AHD) and referred to Apollo Hospitals Dhaka.

On arrival in AHD ER, she was conscious, oriented, mildly anemic, and severely icteric, no pedal edema, and stable hemodynamics. 
Obstetrical examination revealed fundal height 36 weeks, singleton pregnancy with cephalic presentation. Fetal movement and fetal heart rate was satisfactory. From the very beginning she has been managed by a multi-disciplinary team (obstetrician, gastroenterologist, intensivist, anesthesiologist, haematologist). Thorough investigations revealed Haemoglobin $11.3 \mathrm{gm} / \mathrm{dl}$, TLC-20,000/cmm, Total platelet count $1,80,000 / \mathrm{cmm}$ with neutrophilic leucocytosis; S. Electrolytes-Normal, S. Creatinine-1.46mg/dl, deranged LFT(Serum Bilirubin-16.5mg/dl, S.Albumin-2.0 gm/dl) and DIC profile(INR-2.00, APTT-61sec, D-Dimer $>4400 \mathrm{~g} / \mathrm{L})$, BT/CT- Normal, S. Uric acid-7.5mg/dl. All viral markers were negative. Feto-maternal risk benefit was critically assessed and decision taken for termination of pregnancy while keeping arrangements for management of possible complications for surgery and systemic derangement. She delivered a male baby by C-section on 27/11/2011 at 02:30 PM under G/A with smooth post operative recovery. But after 3 hours of operation, she developed abdominal distension with large hemorrhagic collection in drain tube that was kept in peritoneal cavity but there was no PPH. Patient was shifted to SICU for close monitoring. Gradually abdominal distension was increased with huge drain tube collection. Patient was managed with blood and blood products transfusion. She received 5 units of whole blood, 1 unit PRBC \& 4 units of FFP on that day. USG abdomen revealed large abdomino-pelvic hemorrhagic collection. Decision was taken for re-exploration on 28.11.11 by combined team (general surgeon \& obstetrician). As bleeding continued, blood transfusion was considered with parallel monitoring of CBC, Coagulation profile. During re-exploration huge amount of clotted \& small amount of fresh blood was found in abdominal cavity and suctioned out. Liver bed injury was excluded by general surgeon. Uterus was found flabby but no bleeding from uterine wound.
Total abdominal hysterectomy was done, abdomen closed with three drains in situ (one subhepatic, one in peritoneal cavity \& another in sub-rectal space). Then pt was shifted to SICU with mechanical ventilator support. On that day, patient received total 7 units of whole blood, 8 units of FFP \& 2 units of platelet concentration. But still from the next day of exploratory laparotomy \& TAH, continuous draining of fresh blood through all drain tubes approximately 2.5-3.5L/day of which 2/3rd coming through Subhepatic drain. On 29.11.11 patient received 2 units of PRBC, 4 units of FFP \& 2 units of Platelet concentrate. As bleeding continued, patient was receiving blood \& blood products daily according to parameters \& investigation reports. She was maintained on mechanical ventilator, stable hemodynamics without ionotrope support, good renal function with adequate urine output, Chest skiagram-bilateral basal consolidation, improving LFT \& coagulation profile. She was in hepatic encephalopathy as serum ammonia was high. Again pt developed abdominal distension with moderate hemorrhagic collection in DT that was kept in peritoneal cavity. Abdomino-aorto angiogram was done to elicit the bleeding point and suggested features of chronic liver disease (hepatosplenomegaly and portosystemic collaterals), cholelithiasis, large abdominopelvic hematoma with active bleeding in the pelvis through portosystemic collaterals. Duplex left collecting system with left hydroureteronephrosis - likely due to compression of left ureter by pelvic hematoma. Bilateral basal lung consolidation. CT-scan of whole abdomen revealed large haematoma within pelvic cavity with upward extension on sides, moderate ascites, and mild fatty infiltration of liver with sludge within gall bladder with mild splenomegaly.

So finally again patient was taken to OT on 05.12.11 along with general surgeon but no bleeding source was found. All previous drain 
tubes were removed and a new drain tube kept in situ along with surgical mops over the bleeding area intra-abdominally. Till that day, patient received total 18 units Whole blood, 12 units PRBC, 38 units FFP \& 24 units Platelet conc. From the next day, drain tube collection was reduced significantly and again pt was re-opened on 08.12.11 for removal of mops \& drain tube. After that intervention, patient was on mechanical ventilator with weaning mode, haemodynamically stable, reduced abdominal girth, and improved other parameters also. But she was having thrombocytopenia and managed with platelet transfusion. She was extubated on 09.12.11 evening uneventfully, maintaining Spo2 well on room air though a bit drowsy \& lethargic. Upto 10.12.2011 she received total 18 units whole blood, 12 units PRBC, 40 units FFP, 36 units of platelet conc.

Gradually patient's general condition improved slowly and shifted out of ICU to general bed, continued with all supportive and symptomatic management. Meanwhile she received 2 units of whole blood, 5 units of PRBCs and 4 units of platelet concentrate accordingly. She was discharged on 12.01.2012 walking on her own feet.

\section{Discussion}

Despite significant controversy, several massive blood transfusion protocols have been developed to simplify and standardize transfusion practices. A 1:1:1 ratio of packed RBCs to fresh frozen plasma to platelet concentrations to avoid dilutional and consumptive coagulopathy and thrombocytopenia. ${ }^{13}$ This is a more physiological regimen similar to whole blood transfusion and called haemostatic resuscitation focuses on the early correction of coagulopathy that is thought to be associated with improved survival. Trauma exsanguinations protocol, an immediate release of blood products in a predefined ratio of 10 units of PRBCs to 4 units of FFP to 2 units of platelets followed by continued release of 6 units of PRBCs to 4 units of FFP to 2 units of single donor platelets thereafter confirmed a 74\% reduction in mortality and total blood product consumption was also significantly reduced. ${ }^{14}$ Transfusion Packages, 5 units of PRBCs, 5 units of FFP and 2 Platelet concentrates to improve haemostatic competence.

In our case study, patient required massive transfusion though we couldn't follow any of the above protocol strictly but we tried our best with 2:1:1 or 1:1.5: 1 and so on. Despite this, the patient was in minimal coagulopathy or very low grade DIC, there was also no significant thrombocytopenia. With successful management, she neither went to any hypovolumic / cardiogenic shock nor required any ionotropic support any time, no other sites of active bleeding.

Patients who have sustained severe hemorrhage and require massive transfusion commonly have potential life threatening complications. An early and profound coagulopathy with dilutional thrombocytopenia. $^{13}$ Traditional resuscitation techniques using large amounts of crystalloid and PRBC without other blood products can exacerbate this coagulopathy. ${ }^{15}$ Most common cause of bleeding following a large volume transfusion is dilutional thrombocytopenia. This should be suspected and treated first before moving on to factor deficiencies.

Citrate toxicity results when the citrate in the transfused blood begins to bind calcium in the patient's body. Clinically significant hypocalcaemia does not usually occur unless the rate of transfusion exceeds one unit every five minutes or so. Treatment is with intravenous calcium administration. Citrate metabolism is primarily hepatic, so hepatic dysfunction or disease can cause this effect to be more pronounced. Though our patient had profound hepatic 
dysfunction, citrate toxicity was prevented with regular and appropriate intravenous administration of calcium.

Hypothermia during massive blood transfusion is an absolute indication for the warming of all blood and fluids to body temperature as it is being given and we followed this protocol strictly to minimize the chances to develop.

Acid-Base disturbances: most common abnormality is a metabolic alkalosis. Patients may initially be acidotic because the blood load itself is acidic and there may be a prevailing lactic acidosis from hypoperfusion. However, once normal perfusion is restored, any metabolic acidosis resolves and the citrate and lactate are then converted to bicarbonate in the liver. As the hepatic function gradually improved and the patient never developed renal dysfunction and was on mechanical ventilator, so acid-base disturbances was handled very easily.

Serum Potassium can rise as stored blood is given. The potassium concentration in stored blood increases steadily with time. So we tried our best to transfuse fresh blood components.

TRALI, transfusion related acute lung injury is now the leading cause of transfusion-associated mortality, even though it is under diagnosed \& underreported. $^{16-18}$ It is a serious blood transfusion complication characterized by the acute onset of non-cardiogenic edema following transfusion of blood products occurring during or within 6 hours after a transfusion. It is typically associated with plasma components such as Platelets and Fresh Frozen Plasma though cases have been reported with packed red blood cells since there is some residual plasma in the packed cells.

ARDS, Acute respiratory distress syndrome is another life threatening lung condition and should be managed in ICU requiring ventilator support. However high FFP/PRBC ratio is associated with a $52 \%$ lower risk of mortality but on the other hand high FFP/PRBC ratio is associated with a two-fold higher risk of ARDS. Sperry et al. ${ }^{19}$ In our case, patient developed features of early ARDS or TRALI after few initial transfusion and was managed successfully with mechanical ventilator support.

In patients with critical bleeding requiring massive transfusion, temperature, acid-base status, ionized calcium, hemoglobin, platelet count, Coagulation/DIC profile, fibrinogen level should be measured early and frequently. Values indicative of critical physiologic derangement include: temperature $<35^{\circ} \mathrm{C}$, $\mathrm{pH}<7.2$, base excess $>-6$, lactate $>4 \mathrm{mmol} / \mathrm{L}$, ionized $\quad$ calcium $<1.1 \mathrm{mmol} / \mathrm{L}$, Platelet count $<50,000 / \mathrm{cmm}, \mathrm{PT}>1.5 \mathrm{x}$ normal, INR $>1.5$, APTT $>1.5 \mathrm{x}$ normal, fibrinogen level $<1.0 \mathrm{gm} / \mathrm{L}^{20}$

With successful treatment, values should trend towards normal. Once definitive control of hemorrhage has been established, a restrictive approach to blood transfusion should be implemented. Guidelines for transfusion in the trauma patient have been established as a standard operating procedure to guide PRBC transfusion therapy for critically ill patients after the immediate resuscitation phase and to minimize the adverse consequences of potentially unnecessary transfusion. This protocol considers that the acute hemorrhage has been controlled, the initial resuscitation has been completed, and the patient is stable in ICU with no evidence of ongoing bleeding. This guideline advocates a trigger for PRBC transfusion of haemoglobin of $<7 \mathrm{gm} / \mathrm{dl}$ (haematocrit $<21 \%$ ). ${ }^{21}$

Mortality is high in massive transfusion and its etiology is multifactorial. The lethal triad of acidosis, hypothermia \& coagulopathy has the 
highest mortality. The acidosis and hypothermia is managed with ease but the coagulopathy is critically difficult to correct.

\section{Conclusion}

Though massive blood transfusion is life saving to severely injured/traumatized patient or actively bleeding patient, a standard protocol should be followed with highest precaution. Thus we can reduce the associated potential complications and ultimately reduce the morbidity and mortality significantly. In patients with critical bleeding requiring massive transfusion, hemoglobin concentration should be interpreted in the context of hemodynamic status, organ perfusion and tissue oxygenation. The use of a massive transfusion protocol to facilitate timely and appropriate use of PRBC and other blood components may reduce the risk of mortality and ARDS. To achieve this goal, the critically bleeding patient should be managed in ICU with multi-disciplinary approach in a single set-up.

\section{References}

1. Harvey MP, Greenfield TP, Sugrue ME. Massive blood transfusion in a tertiary referral hospital: clinical outcomes and haemostatic complications. Med J Aust. 1995;163:356-359.

2. Hearnshaw S, Travis S, Murphy M. The role of blood transfusion in the management of upper and lower intestinal tract bleeding. Best Pract Res Clin Gastroenterol. 2008;22:355-371.

3. Forcione DG,Alam H B, Klava SP. Case records of the Massachusetts General Hospital: case 9-2009; an 81 year old man with massive rectal bleeding. N Eng J Med. 2009;360:1239-1248.

4. Maltz GS, Siegel JE, Carson JL. Hematological management of gastrointestinal bleeding. Gastroenterol Clin North Am. 2000;29:169-187.

5. Piastra M, Di Rocco C, Tempera A. Massive blood transfusion in choroids plexus surgery:10 years experience. J Clin Anesth. 2007;19:192-197.

6. Kozek- Langenecker S. Management of massive operative blood loss. Minerva Anesthesiol.
2007;73:401-415.

7. Erber WN. Massive blood transfusion in the elective surgical setting. Transfus Apher Sci. 2002;27:83-92. 8. Lim RC, Olcott C, Robinson AJ. Platelet response and coagulation changes following massive blood replacement. J Trauma. 1973;13:577-582.

9. Malone DL Hess JR, Fingerhut A. MT practices around the globe and a suggestion for a common MT protocol. J Trauma. 2006;60:S91-S96.

10. Mollison PL, Engelfreit CP, Contreras M. Transfusion in oligaemia; blood transfusion in clinical medicine. 11th ed. Oxford, UK : Blackwell Scientific Publications. 2005;47.

11. Levy JH. MT coagulopathy. Semin Hematol. 2006;43:S59-S63.

12. Gonzalez EA, Moore FA, Holcomb JB. Fresh frozen plasma should be given earlier to patients requiring MT. J Trauma. 2007;62:112-119.

13. MacLeod JB, Lynn M, McKenney MG. Early coagulopathy predicts mortality in trauma. J Trauma. 2003;55:39-44.

14. Cotton BA, Gunter OL, Isbell J. Damage control hematology: the impact of a trauma exsanguinations protocol on survival and blood product utilization. J Trauma. 2008;64:1177-1182.

15. Hardy JF, DeMoerloose P, Samama CM. The coagulopathy of MT. Vox Sang. 2005;89:123-127.

16. Toy P Popovsky MA, Abraham E. Transfusion-related acute lung injury: defined and review. Crit Care Med. 2005;33:721-726.

17. Gajic E, Rana R, Winters JL. Transfusion-related acute lung injury in the critically ill: prospective nested case-control study. Am J Respir Crit Care Med. 2007;176:886-891.

18. Rubenfeld GD, Caldwell E, Peabody E. Incidence and outcomes of acute lung injury. N Eng J Med. 2005;353:1685-1693.

19. Sperry JL, Ochoa JB, Gunn SR. An FFP:PRBC transfusion ratio $>1: 1.5$ is associated with lower risk of mortality after MT. J Trauma. 2008;65:986-993.

20. National blood Authority. [cited 2011 Jun30] http://www.nba.gov.au.

21. West MA, Shapiro MB, Nathens AB. Inflammation and the host response to injury, a large-scale collaborative project: patient-oriented research core-standard operating procedures for clinical care; IV . Guidelines for transfusion in the trauma patient. J Trauma. 2006;61:436-439. 